\title{
Initial Results from a Pilot Comparative Effectiveness Study of 3 Methotrexate-based Consensus Treatment Plans for Juvenile Localized Scleroderma
}

\author{
Suzanne C. Li ${ }^{\mathbb{D}}$, Kathryn S. Torok (D), C. Egla Rabinovich, Fatma Dedeoglu ${ }^{\mathbb{D}}$, Mara L. Becker, \\ Polly J. Ferguson (D), Sandy D. Hong, Maria F. Ibarra, Katie Stewart, Elena Pope (D), \\ Gloria C. Higgins, Ronald M. Laxer, Thomas Mason II ${ }^{\mathbb{D}}$, Robert C. Fuhlbrigge (D), \\ and Tracy Andrews ${ }^{\mathbb{D}}$, for the CARRA Registry Investigators
}

ABSTRACT. Objective. To perform a comparative effectiveness feasibility study in juvenile localized scleroderma (LS), using standardized treatment regimens (consensus treatment plans; CTP).

Methods. A prospective, multicenter 1-year pilot observational cohort study was performed by Childhood Arthritis and Rheumatology Research Alliance (CARRA) LS workgroup members. Patients with active, moderate to severe juvenile LS were treated with one of 3 CTP: methotrexate alone, or in combination with intravenous $(30 \mathrm{mg} / \mathrm{kg} / \mathrm{dose}$ for $3 \mathrm{mos})$ or oral corticosteroids $(2 \mathrm{mg} / \mathrm{kg} /$ day tapered by 48 weeks).

Results. Fifty patients, with demographics typical for juvenile LS, were enrolled, and 44 (88\%) completed the study. Most had extracutaneous involvement. Patients improved in all 3 CTP, with $>75 \%$ having a major or moderate level of improvement compared to baseline. Damage accrued in some patients. Major deviations from prescribed regimen resulted from medication intolerance $(n=6 ; 14 \%)$ or treatment failure $(n=11 ; 25 \%)$; failures occurred in all 3 CTP. Significant responses to treatment were demonstrated by LS skin scoring measures and overall physician assessments, with differences in response level identified in some patient subsets. Response differences were associated with baseline disease activity level, LS subtype, skin disease extent, and extracutaneous involvement.

Conclusion. This study demonstrates the feasibility of conducting juvenile LS comparative effectiveness studies. The CTP were found to be safe, effective, and tolerable. Our assessments performed well. Because damage is common and may progress despite effective control of activity, we recommend initial treatment efficacy be evaluated primarily by activity measures. Potential confounders for response were identified that warrant further study. (First Release May 15 2020; J Rheumatol 2020;47:1242-52; doi:10.3899/jrheum.190311)

Key Indexing Terms:

PEDIATRIC RHEUMATIC DISEASES

SCLERODERMA

METHOTREXATE

CLINICAL TRIALS

\section{DISEASE ACTIVITY SCORE}

From the Joseph M. Sanzari Children's Hospital, Hackensack University Medical Center, Hackensack Meridian School of Medicine at Seton Hall University, Nutley, New Jersey; UPMC Children's Hospital of Pittsburgh, Pittsburgh, Pennsylvania; Duke University School of Medicine, Durham, North Carolina; Boston Children's Hospital, Boston, Massachusetts; Children's Mercy Hospital, Kansas City, Missouri; University of Iowa Carver College of Medicine; University of Iowa Stead Family Children's Hospital, Iowa City, Iowa; Children's Medical Center of Dallas and UT Southwestern, Dallas, Texas; The Ohio State University, Columbus, Ohio; Mayo Clinic, Rochester, Minnesota; University of Colorado-Denver and Children's Hospital Colorado, Denver, Colorado; Rutgers University, School of Public Health, Newark, New Jersey, USA; University of Toronto and The Hospital for Sick Children, Toronto, Ontario, Canada.

The Childhood Arthritis and Rheumatology Research Alliance (CARRA) Legacy Registry was supported by a grant from the US National Institute of Arthritis and Musculoskeletal and Skin Diseases of the National Institutes of Health (NIH) under award number RC2AR058934. The content is solely the responsibility of the authors and does not necessarily represent the official views of the NIH. CARRA, Friends of CARRA, the Arthritis Foundation, and the Duke Clinical Research Institute also supported the CARRA Legacy Registry. This study was funded primarily by an innovative research grant from the Arthritis foundation (PI: SL). CARRA provided additional funding for data analysis (publication grant, PI: SL) and in-kind resources to support management and maintenance of the registry for the study, online meetings, and face-to-face meetings at annual meetings of CARRA and the American College of Rheumatology. Support for biobanking was provided by independent funding from The Nancy Taylor Foundation for Chronic Diseases Inc. (PI: KST, Children's Hospital of Pittsburgh Pediatric Scleroderma Fund).

S.C. Li, MD, PhD, Joseph M. Sanzari Children's Hospital, Hackensack University Medical Center, Hackensack Meridian School of Medicine at Seton Hall University; K.S. Torok, MD, UPMC Children's Hospital of Pittsburgh; C.E. Rabinovich, MD, MPH, Duke University School of Medicine; F. Dedeoglu, MD, Boston Children's Hospital; M.L. Becker, MD, MSCE, Children's Mercy Hospital; P.J. Ferguson, MD, University of Iowa Carver College of Medicine; S.D. Hong, MD, University of Iowa Stead Family Children's Hospital; M.F. Ibarra, MD, Children's Mercy Hospital; K. Stewart, MD, Children's Medical Center of Dallas and UT Southwestern; E. Pope, MD, University of Toronto and The Hospital for Sick Children; G.C. Higgins, $M D, P h D$, The Ohio State University; R.M. Laxer, MD, University of Toronto and The Hospital for Sick Children; T. Mason II, MD, Mayo Clinic; R.C. Fuhlbrigge, MD, PhD, University of Colorado-Denver, and Children's Hospital Colorado; T. Andrews, MA, Rutgers University, School of Public Health.

Address correspondence to Dr. S.C. Li, Hackensack University Medical Center, Imus PC337, 30 Prospect Ave., Hackensack, New Jersey 07061, USA.E-mail: suzanne.li@hackensackmeridian.org

Accepted for publication September 30, 2019.

Personal non-commercial use only. The Journal of Rheumatology Copyright $(\subset) 2020$. All rights reserved. 
Localized scleroderma (LS), which includes circumscribed morphea and linear scleroderma, is an autoimmune disease characterized by inflammation and fibrosis ${ }^{1,2}$. It is the most common childhood form of scleroderma, and pediatric onset has worse morbidity than adult disease ${ }^{3,4}$. Morbidity includes uveitis, seizures, arthropathy, and growth disturbances such as limb and facial hemiatrophy. Functional impairment is found in $27-56 \%$ of juvenile LS patients $5,6,7,8,9$.

Treatment focuses on controlling inflammation, because effective treatment options for fibrosis are limited. Most North American and European pediatric rheumatologists, and many other physicians, agree on the use of methotrexate (MTX) to treat moderate to severe disease $\mathrm{e}^{10,11,12,13,14}$. A single randomized, double-blind, placebo-controlled study evaluating the efficacy of MTX in 70 patients with juvenile LS who received an initial oral corticosteroid (CS) course demonstrated a higher response rate among patients in the MTX compared to the placebo arm (67.4\% vs $29.2 \%$, respectively $)^{15}$. Many case reports also support MTX's efficacy $^{16-22}$. However, physicians differ regarding MTX dosing, route, and duration, the need for CS, and the CS regimen ${ }^{10,23}$. In a survey of North American pediatric rheumatologists asking their preference for treating juvenile LS with a standardized MTX-based regimen, $31 \%$ preferred MTX monotherapy, 36\% MTX with intravenous pulse CS, and 23\% MTX with oral $\mathrm{CS}^{23}$. Given the lack of data to support the superiority of 1 regimen over another, there is clearly a need for comparative effectiveness studies in juvenile LS.

To assess feasibility and methodology for comparative effectiveness studies in juvenile LS, we evaluated the safety and tolerability of 3 MTX-based regimens (consensus treatment plans; CTP) ${ }^{23}$ in a pilot 1-year open-label study of 50 patients initiating treatment for active juvenile LS. We identified the frequency of and reasons for deviation from the CTP, adverse events (AE) associated with therapies, and response to treatment by several clinical assessments.

\section{MATERIALS AND METHODS}

Study protocol. The LS workgroup of the Childhood Arthritis and Rheumatology Research Alliance (CARRA) conducted this study; details on the development of the study and CTP choice are described elsewhere ${ }^{24}$. Each participating site $(\mathrm{n}=10)$ obtained institutional ethics approval for both the study itself and the informed consent form, which included our intent to publish the results of the study and measures to protect confidentiality. The written consent was signed by either the patient or by the patient's parent/guardian with written or verbal assent of the patient, as appropriate for age. Deidentified data were analyzed at Hackensack University Medical Center, the coordinating center, under ethics approval number Pro00001481.

Inclusion and exclusion criteria for participation in the study are shown in Table $1^{23,25,26}$. At entry, patients started treatment with 1 of 3 MTX-based CTP (Figure 1A): MTX monotherapy (CTP A, $1 \mathrm{mg} / \mathrm{kg} / \mathrm{week}$, maximum $25 \mathrm{mg}$; same dose for all CTP), MTX with intravenous (IV) CS (CTP B; IV CS $30 \mathrm{mg} / \mathrm{kg} / \mathrm{dose}$, maximum $1000 \mathrm{mg}$, either 3 consecutive days/month $\times 3$ months or $1 / \mathrm{wk} \times 12$ weeks), or MTX with oral CS (CTP C; prednisone or prednisolone $2 \mathrm{mg} / \mathrm{kg}$ /day, maximum $60 \mathrm{mg}$, divided bid, tapered to $1 \mathrm{mg} / \mathrm{kg} / \mathrm{d}$ by 8 weeks, $0.5 \mathrm{mg} / \mathrm{kg} / \mathrm{d}$ by 16 weeks, $0.25 \mathrm{mg} / \mathrm{kg} / \mathrm{d}$ by 24 weeks, and off by 48 weeks). Subcutaneous administration of MTX was recommended. Choice of CTP was decided by treating physician and the patient's family (discussed by $\mathrm{Li}$, et $a l^{24}$ ). Patients were monitored at 6 study visits over 1 year: baseline, 2, 4, 6, 9, and 12 months, with recommended visit windows of \pm 1 month. At the initial visit, demographic information was collected, including subtype as defined by Padua criteria ${ }^{24}$, medical history, treatment history for juvenile LS, and family history. At subsequent visits, medication history and $\mathrm{AE}$ were recorded. $\mathrm{AE}$ were graded according to the Common Terminology Criteria ${ }^{27}$. Laboratory studies were done at the discretion of the treating physicians. Most of the data were entered into a Web-based registry (i2b2 CARRA Legacy Registry); remaining data were recorded in a database at the principal investigator's (PI) site. There was an optional biorepository substudy, banking blood samples for future studies.

The same physician evaluated a given patient at all study visits to avoid interrater variability. Evaluations included modified localized skin severity index (mLoSSI), LS Damage index (LoSDI), and measures we recently developed ${ }^{23,28,29}$. The mLoSSI divides the body into 18 anatomic sites for scoring, and is calculated as the sum of disease extension (scored 3 if present), erythema (scored $0-3$, severe), and skin thickening of the lesion edge (scored 0-3, severe) at all affected $\operatorname{sites}^{28,30}$. Building from the mLoSSI, the LS Cutaneous Activity Measure (LSCAM) also scores additional variables that were found associated with activity in another study ${ }^{31}$. LSCAM is calculated as the sum of disease extension, erythema, maximum lesion skin thickening, violaceous color, tactile warmth, and waxy white or yellow; erythema and skin thickening are scored 0 to 3 (severe), the other variables 0 or 1 if present ${ }^{23}$. The LoSDI and LS Cutaneous Damage Measure (LSDam) sum dermal atrophy, subcutaneous atrophy, and dyspigmentation across affected sites $^{29}$. The LSDam also scores maximum lesional skin thickening. Variables in LoSDI and LSDam are scored 0 to 3 (severe), with scoring examples provided in LS Scoring Atlas ${ }^{23}$.

Extracutaneous involvement (ECI) considered secondary to LS was scored as 0 or 1 if present; the list of scored items was generated based upon literature ${ }^{23,32,33}$. Joint involvement included arthritis (joint swelling) and contractures (limited range of joint motion without swelling). Growth difference was scored if the clinician considered it to be obvious and significant, and included limb girth and length differences, and facial and truncal hemiatrophy. Skin activity scoring was performed at all visits, while skin damage and ECI scoring were assessed at visits 0, 6, and 12 months. Efforts to standardize scoring are described elsewhere ${ }^{24}$.

Physician's global assessment of disease activity (PGA-A) and physician's global assessment of disease damage (PGA-D) were scored on 0-10 (high) Likert scales. PGA-A was scored at every visit; PGA-D at 0-, 6-, and 12 -month visits. Physician assessment of overall disease status compared to baseline visit ( $\Delta$-disease status), and physician assessment of overall activity status compared to baseline visit ( $\Delta$-activity status) were scored on a 7-level Likert scale from major improvement (3) to major worsening $(-3)$ at the last visit. These global assessments included consideration of extracutaneous involvement. No guidelines were provided on scoring these assessments; instead, each study investigator determined how to score them based upon their clinical evaluation and judgment. Patients and/or their parents were asked to complete health-related quality of life assessments at visits 0,6 , and 12 months $^{34,35,36,37,38}$.

Protocol deviations. Changing the route of MTX administration, having a temporary reduction or lapse in taking MTX ( $<2$ weeks), and missing a prednisone taper target because of a delayed study visit were considered minor deviations. Actions considered major deviations were stopping treatment (by physician, patient, or family), prolonged change in specified dose or duration of CTP medication(s), and/or using an immunomodulator not specified by initial CTP. Patients who withdrew or failed to followup were scored as dropouts and not included in response analysis. Treatment failure (TF) was defined as inadequate response to the initial regimen leading to treatment with additional CS and/or non-CTP-specified immunomodulator. Data analysis. Data were summarized using frequencies (\%) for categorical variables and median [interquartile range (IQR)] for continuous variables. Patient response was analyzed based upon intent to treat and

Personal non-commercial use only. The Journal of Rheumatology Copyright $\subset$ 2020. All rights reserved. 
Table 1. Juvenile localized scleroderma CTP pilot study entry criteria: inclusion, exclusion, and active disease criteria.

Inclusion criteria

1. Localized scleroderma diagnosed by a pediatric rheumatologist or pediatric dermatologist according to Padua Preliminary

Classification criteria; these criteria exclude eosinophilic fasciitis ${ }^{24}$

2. Fulfill active disease criteria, listed below

3. Moderate to severe disease that warrants systemic therapy in the opinion of the treating physician

a. Includes all subtypes that involve deeper tissue(s), extensive skin involvement, and/or extracutaneous involvement.

4. Age $<18$ yrs at onset of disease

5. Age $<21$ yrs at onset of treatment

Exclusion criteria

1. Treated with systemic corticosteroids in the prior 2 weeks

2. Treated with methotrexate or mycophenolate mofetil within the prior 3 months

3. Another defined systemic rheumatic disease (e.g., systemic sclerosis)

Active disease criteria

Intolerance to study medications

Group 1: one item suffices

1. New lesion(s) within the prior 3 months, documented by clinician

2. Extension of an existing lesion within the prior 3 months, documented by clinician

a. Lesion extension observed in serial photographs or tracings, or detecting $\geq 30 \%$ difference in lesion size (max length $\times$ width)

3. Documentation of active or progressive deep tissue involvement

a. Can be by clinical examination, photographs, MRI, or ultrasound

4. Erythema of moderate or severe level in lesion or an erythematous lesion border

a. Rating of erythema scoring level based upon LS Scoring Atlas

5. Violaceous lesion or border color

Group 2: need at least 2 items

1. Patient or parent report of new lesion OR extension of existing lesion occurring within the prior 3 months

a. This criterion ONLY applies for new patients (i.e., first visit to clinician's office)

2. Mild erythema of lesion

a. Rating of erythema scoring level based upon LS Scoring Atlas

3. Severe or moderate induration of lesion border

a. Assessed according to modified Rodnan skin score levels ${ }^{25}$

4. Tactile warmth of the lesion

a. Examiner appreciation of temperature difference based upon comparison to control site (unaffected contralateral site if available)

5. Worsening hair loss in scalp, eyebrow, or eyelashes; documented by clinician

6. Elevated creatine kinase level in the absence of other source

7. Lesion biopsy showing active disease (based upon pathologist report). Typically, would be presence of lymphocytes, plasma cells, eosinophils, or other white blood cells

These criteria were previously developed ${ }^{23}$ for the purpose of directing comparative effectiveness studies in juvenile LS and were not intended to qualify or disqualify patients for any specific treatment. The LS Scoring Atlas was generated by the LS workgroup of CARRA and contains photographs of patient lesions demonstrating the different visible scored features; $\geq 80 \%$ consensus agreement by work group members was required for the photograph to be included in the atlas $^{23}$. Suggested modifications of these entry criteria for future studies are described elsewhere ${ }^{24}$. LS: localized scleroderma; CTP: consensus treatment plan; CARRA: Childhood Arthritis and Rheumatology Research Alliance; MRI: magnetic resonance imaging.

censored for TF. Comparisons across groups, where appropriate, were performed using chi-square or Fisher's exact tests for categorical data and Wilcoxon rank-sum tests or sign-rank tests for continuous, nonparametric data. Normality was assessed using the Shapiro-Wilks test $(\mathrm{p}<0.001)$. Spearman correlation was performed to examine correlations between activity, damage, and improvement in scores across visits, as well as lesion and disease characteristics. We used the most recent non-missing data when comparing activity or damage scores to a previous visit. An alpha of 0.05 was used to assess significance; given the small sample, we also noted variables with a $\mathrm{p}$ value $<0.1$. Data were analyzed using SAS version 9.4 (SAS Institute Inc.).

\section{RESULTS}

Patient characteristics. Patients were enrolled into all 3 CTP, achieving the target enrollment of 50 patients (Figure 1). Most patients were white $(46 ; 92 \%)$ and had linear scleroderma subtype $(30 ; 60 \%)$. The median age of disease onset was 9.6 years (IQR 6.1-11.7), and disease duration
13 months (IQR 6-53.8). Forty-one patients (82\%) were newly diagnosed and naive to systemic immunosuppressants; the other 9 patients had a disease relapse off prior systemic treatment. Thirty-seven patients $(74 \%)$ had ECI, primarily growth difference $(23 ; 46 \%)$ and/or joint involvement $(20 ; 40 \%)$.

Six patients discontinued treatment before the last visit (dropouts, Figure 1A). The remaining 44 patients completed the study and were analyzed for treatment response and AE. CTP groups differed in the number of affected anatomic sites $(\mathrm{p}=0.021)$, and subtype frequency, growth disturbance, and antinuclear antibody (ANA) positivity $(\mathrm{p}<0.1$; Table 2 ). No differences were found for age, disease duration, race, ethnicity, ECI, or family history of autoimmune disease (Table 2, and data not shown).

Safety and tolerability of CTP. Six patients (14\% of patients

Personal non-commercial use only. The Journal of Rheumatology Copyright @ 2020 . All rights reserved 


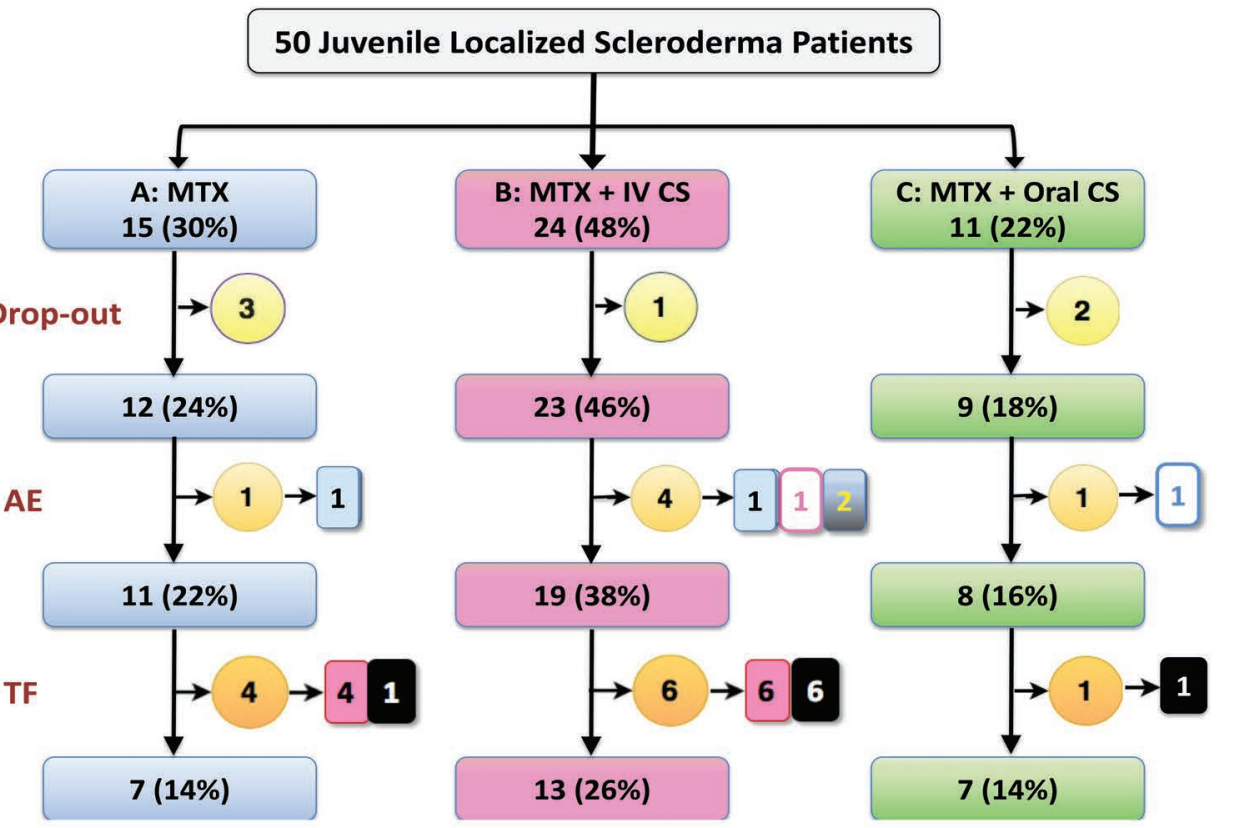

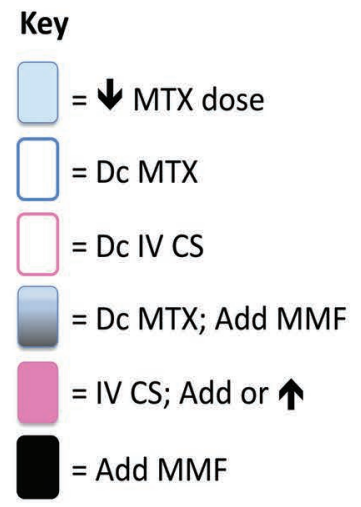

No. in box $=$ no. of patients that followed this change

B
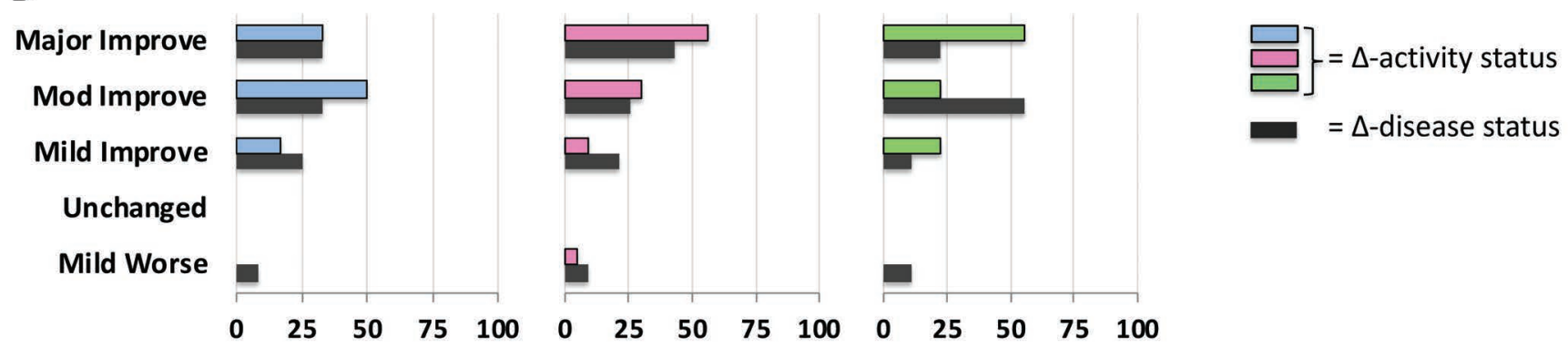

Figure 1. Schematic of the 50 patients who were enrolled in the juvenile LS CTP Pilot Study. A. The 3 CTP are depicted by the blue, pink, and green rectangles: A = MTX monotherapy; B = MTX with IV CS (methylprednisolone, 3 pulse doses per month $\times 3$ months); C $=$ MTX with daily oral CS (prednisone or prednisolone; beginning at $2 \mathrm{mg} / \mathrm{kg} /$ day and tapering off by 48 weeks), respectively. All 3 CTP included the same MTX dose (1 mg/kg/week, maximum $25 \mathrm{mg}$ ); subcutaneous route of administration was preferred. The numbers and percentages in the rectangles refer to the patients enrolled in each CTP, and who remained in their starting CTP after accounting for dropouts, AE that led to a major deviation from the CTP, and TF. The numbers in the circles indicate the number of patients who dropped out, had an AE, or were considered to have TF. Study dropouts occurred in CTP A after the 2-month visit ( $\mathrm{n}=1$ ) or 6-month visit $(n=2)$, in CTP B after baseline visit, in CTP C after baseline visit $(n=2)$. The medication changes related to AE and TF are shown in the boxes to the right of the circles. The numbers in these boxes refer to the number of patients who had this change. B. Bar charts below each CTP indicate the physician assessment of activity status compared to baseline scores ( $\Delta$-activity status, blue, pink, or green bars) or physician assessment of disease status compared to baseline scores ( $\Delta$-disease status, black bars) rating of the 44 patients who completed the last visit based upon intent to treat (excludes dropouts, but includes $\mathrm{AE}$ and TF). The $\mathrm{X}$-axis indicates the percentage of patients who received that rating. AE: adverse event leading to a major deviation from the CTP; CS: corticosteroid; CTP: consensus treatment plan; Dc: discontinue; Improve: improvement; IV: intravenous; jLS = juvenile localized scleroderma; Mod: moderate; MTX: methotrexate; TF: patients judged to have experienced treatment failure by physician, necessitating treatment in addition to that specified by CTP; MMF: mycophenolate mofetil; Worse: worsening.

completing last visit) had a major deviation from the CTP because of medication intolerance (Figure 1A), including 1 (2\%) grade $3 \mathrm{AE}$, a hospitalization for gastroenteritis and dehydration, considered unrelated to the CTP (B). Twenty-one patients (48\%) had a grade $2 \mathrm{AE}$, with no difference in frequency across groups. The most common grade $2 \mathrm{AE}$ were gastrointestinal problems $(\mathrm{n}=11,25 \%)$, which were managed by ondansetron treatment $(n=5)$, changing route of administration $(n=2)$, reducing dose $(n=2)$, or discontinuing MTX $(\mathrm{n}=3)$. Other grade 2 AE were mood problems $(\mathrm{n}=5,11 \%)$, infection $(\mathrm{n}=3,7 \%)$, laboratory abnormalities $(\mathrm{n}=3,7 \%)$, and 1 each of seizure recurrence, hair thinning, lip and nasal ulcer, and blurred vision. One patient discontinued IV CS because of access difficulties. Patients who experienced TF and received additional medication(s) had more grade $2 \mathrm{AE}$ (82\% vs $36 \%$ for non-TF, $\mathrm{p}=0.009)$. Grade $1 \mathrm{AE}$ were more frequent in the oral CS regimen (CTP C, p = 0.005), most commonly weight gain or

Personal non-commercial use only. The Journal of Rheumatology Copyright @ 2020 . All rights reserved. 
Table 2. Demographics of the 44 patients who completed and were receiving treatment at the last study visit.

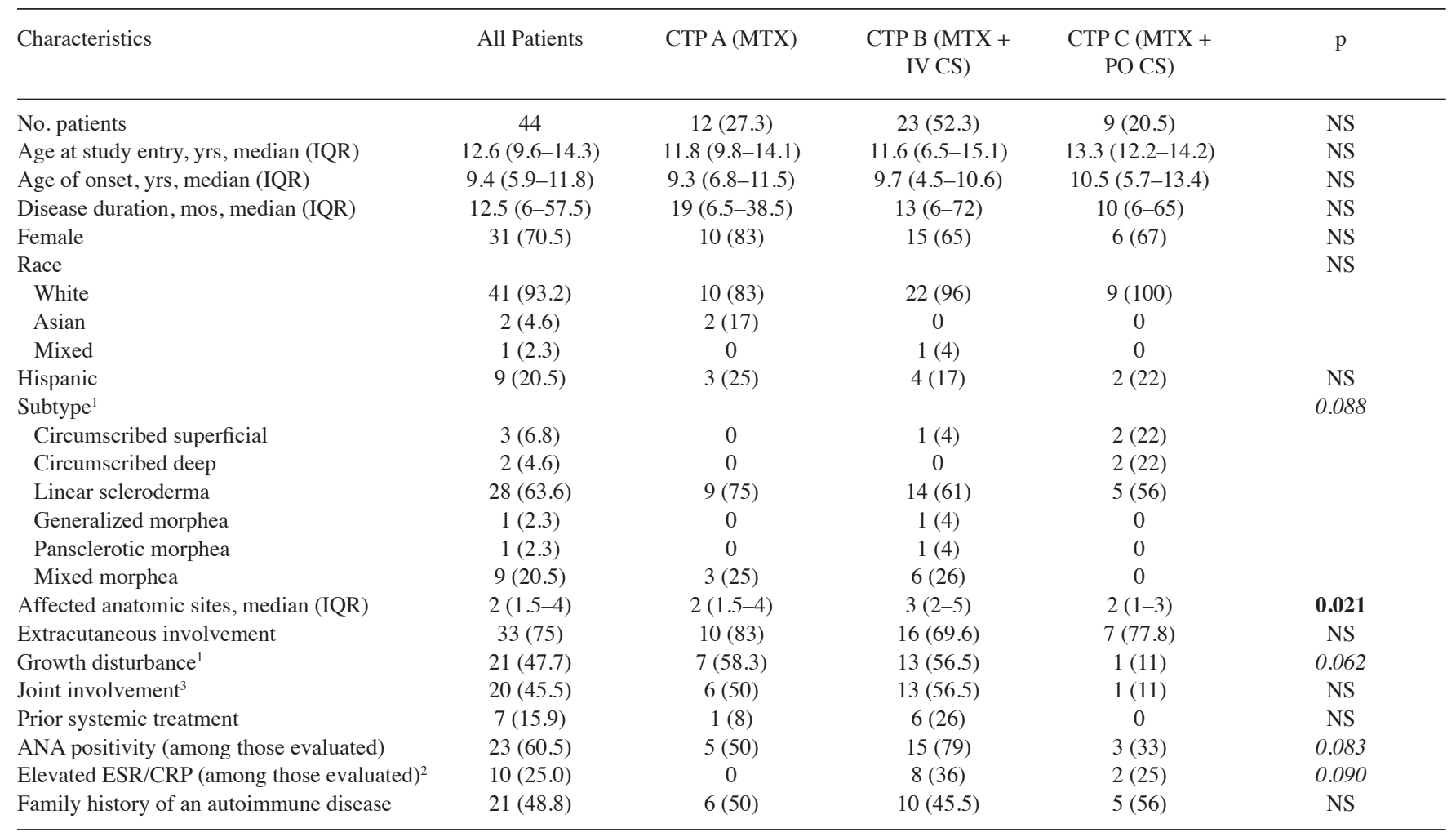

Values are $\mathrm{n}(\%)$ unless otherwise specified. P values for differences across the $3 \mathrm{CTP}$ are shown in last column. Significant $\mathrm{p}$ values are in bold face; $\mathrm{p}$ values that approached significance are in italics. Superscript numbers in first column indicate differences between patients in $1 \mathrm{CTP}$ compared to those in the other 2. Differences between patients in CTP C versus CTP A and B: ${ }^{1} \mathrm{p}<0.05,{ }^{3} \mathrm{p}=0.08$. Differences between patients in CTP C versus CTP A and B: ${ }^{2} \mathrm{p}<0.05$. The last visit was the "12"-month visit for 43 patients, and "9"-month visit for 1 patient. For 2 patients, the chronological time of their "12"-month visit was at 274 or 295 days. We therefore decided to include a subject whose last study visit was the "9"-month visit, which occurred at a chronological time of 283 days. This patient lived far from the study site, did not make the "12"-month visit, but did have a later followup visit. Extracutaneous involvement was scored based on presence at any time during the study. Growth disturbance refers to limb girth or length difference, or facial or trunk hemiatrophy. Joint involvement refers to arthritis (swollen joint) or joint contracture. Other extracutaneous involvement were headache (4), seizure (1), pseudopapilledema (1), Raynaud phenomenon (2), muscle spasm (2), and dental crowding (1). The number of anatomic sites is based upon the divisions used for the LS Cutaneous Activity measure; the body is divided into 19 sites: scalp, face, neck, chest, abdomen, back, buttock, arm (right/left), forearm (right/left), hand (right/left), thigh (right/ left), lower leg (right/left), and foot (right/left). The p values on the "Race" and "Subtype" rows refer to the significant difference found in the distribution of all the listed races or subtypes, respectively. Circumscribed morphea subtype was also found to differ between patients in CTP C versus CTP A and B ( $p=0.004)$. Family history of an autoimmune disease includes rheumatic diseases and additional autoimmune diseases such as celiac disease, type 1 diabetes mellitus, and multiple sclerosis. Thirty-eight patients were evaluated for ANA positivity; 40 patients had an initial ESR or CRP test done. ANA: antinuclear antibody; ESR: sedimentation rate; CRP: C-reactive protein; CTP: consensus treatment plan; IQR: interquartile range; LS: localized scleroderma; NS: nonsignificant; PO: oral; MTX: methotrexate; IV: intravenous; CS: corticosteroids.

Cushingoid features $(\mathrm{n}=13,29.5 \%)$, gastrointestinal problems $(\mathrm{n}=10,23 \%)$, and mood problems $(\mathrm{n}=4,9 \%)$.

Response to CTP. Nearly all patients improved compared to baseline by 2 PGA ( $\Delta$-disease status, $\Delta$-activity status). The $\Delta$-activity status rated 43 of $44(98 \%)$ patients as improved, versus $40(90 \%)$ by $\Delta$-disease status (Figure $1 \mathrm{~B}$ ). Both assessments rated a patient who had disease extension and return of induration at 9 months as worsened. The $\Delta$-disease status rated an additional 3 patients as worsened including 1 with seizure recurrence at 12 months without cutaneous activity signs (linear scleroderma of the head subtype), and 2 who developed more damage features (worsening facial or other growth difference) with no or minimal residual skin disease activity.
While this pilot study was not powered to assess efficacy, we analyzed the response of patients in each CTP to provide information on our assessments and identify potential confounders. PGA-A, mLoSSI, and LSCAM scores decreased from baseline to last visit in all CTP groups when analyzed based upon intent to treat and censored for TF (Table 3). PGA-D, LoSDI, LSDam, and several patient/ parent health-related outcome scores did not differ from baseline to last visit (Table 3).

To track response across the 6 visits, we examined whether and when patients achieved a PGA-A score of 0 . PGA-A $=0$ occurred in $42 \%$ of CTP A, $44 \%$ of CTP B, and $67 \%$ of CTP C patients (Table 4A). When patients with TF were excluded, percentages increased to 50\% (CTP A), 59\% 
Table 3A. Change in cutaneous, physician, and parent/patient scores from baseline to last visit in each CTP based upon intent to treat $(n=44)$.

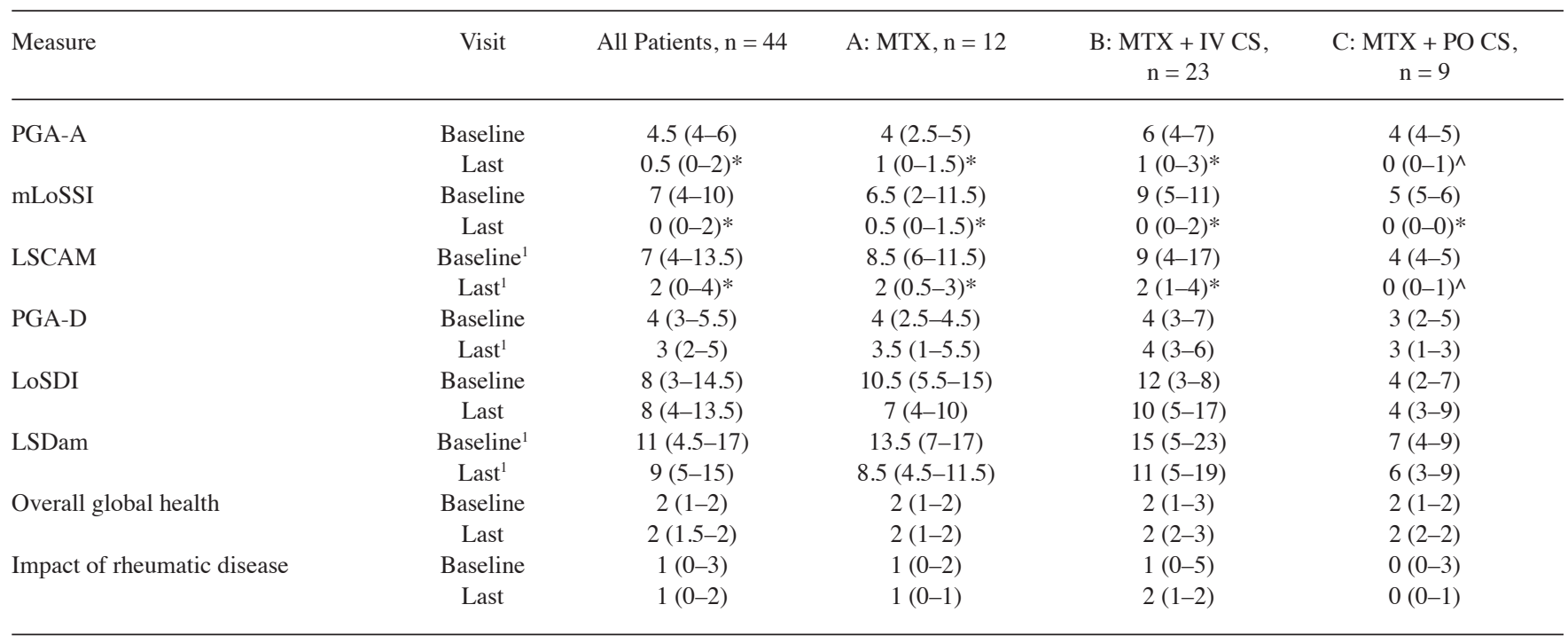

Table 3B. Change in cutaneous, physician, and parent/patient scores from baseline to last visit in each CTP: censored for treatment failures $(\mathrm{n}=33)$.

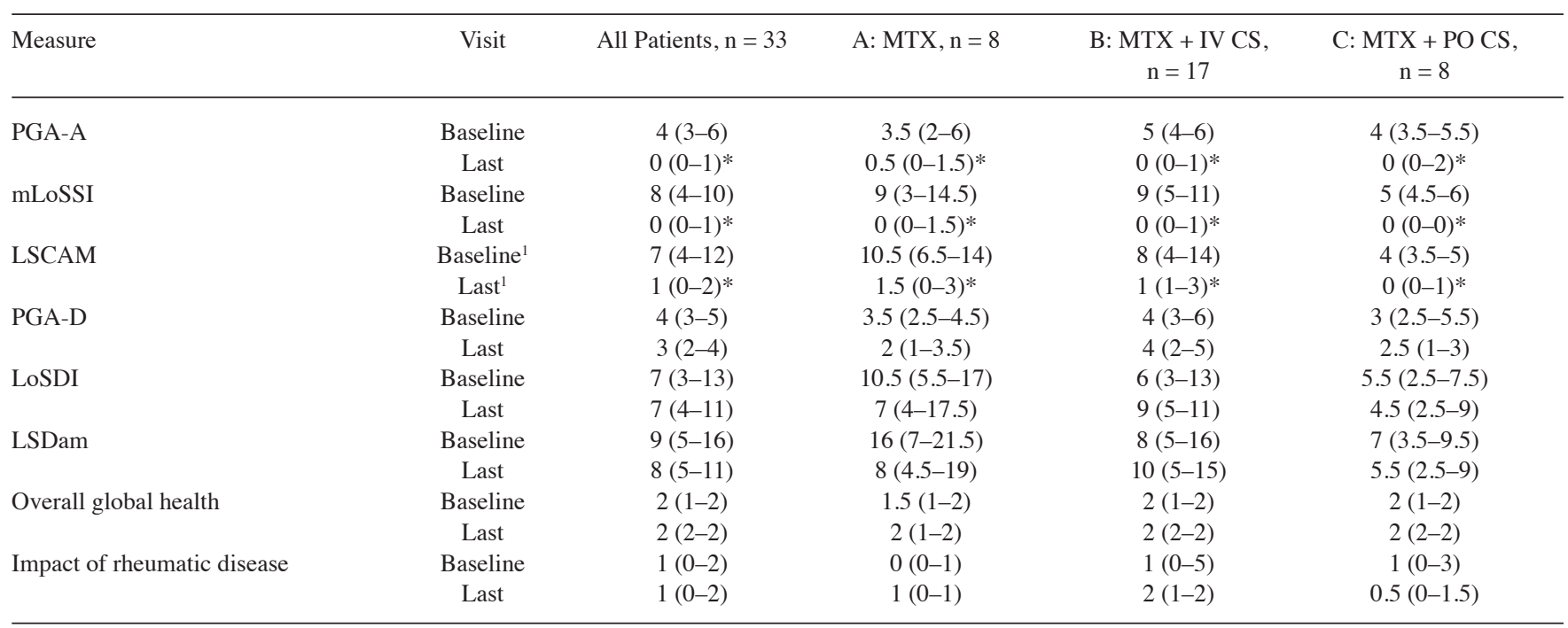

Values shown are median (IQR). Significant changes in scores from baseline to last visit are indicated in All Patients and CTP columns: ${ }^{\wedge} \mathrm{p}<0.01 . * \mathrm{p}<0.001$. Superscript numbers in Visit column indicate differences in scores between patients in CTP C versus those in CTP A and B: ${ }^{1} \mathrm{p}<0.05$. Patients who dropped out or discontinued treatment prior to their 9-month visit $(n=6)$ were not included. Patients who deviated from their initial CTP because of adverse event but were able to continue systemic treatment were included in Table 3A. The mLoSSI and LSCAM score cutaneous activity features, while the LoSDI and LSDam score cutaneous damage features. Overall global health refers to the parent or patient question, "How do you rate your child's health?" (range: excellent $=5$, to very poor $=1$ ). If the child was answering the question, then the question was reworded to focus on the patient's point of view. Effect of rheumatic disease refers to the parent or patient question, "Considering all the ways that your child's rheumatic condition affects your child, rate how your child is doing" $(0=$ no impact, $10=$ highest impact). Additional health-related quality of life measures asked of parents or patients included global pain ("How much pain do you think your child had because of his/her rheumatic condition in the past week?") and the Childhood Health Assessment Questionnaire ${ }^{33}$, neither of which were found to differ significantly between the baseline and last visit. Other collected measures were PedsQL general, PedsQL rheumatology, PedsQL Family Impact, and Children's Dermatology Life Quality Index ${ }^{34,35,36,37}$. CS: corticosteroid; CTP: consensus treatment plan; IQR: interquartile range; IV: intravenous; LoSDI: Localized Scleroderma Damage Index; LSCAM: Localized Scleroderma Cutaneous Activity Measure; LSDam: Localized Scleroderma cutaneous Damage measure; mLoSSI: modified Localized Scleroderma Severity Index; MTX: methotrexate; PedsQL: Pediatric Quality of Life Inventory; PGA-A: physician's global assessment of disease activity; PGA-D: PGA of disease damage; PO: oral.

(CTP B), and 75\% (CTP C; Table 4A). Patients in CTP C (MTX plus oral CS) appeared to reach 0 sooner, but these differences were not significant. Patients in CTP C had lower baseline LSCAM and LSDam scores than patients in CTP A and $\mathrm{B}(\mathrm{p}<0.05$; Table $3 \mathrm{~A})$; mLoSSI or LoSDI scores were not different across groups (Table 3A). The lower cutaneous 
Table 4A. The total number of patients who achieved a PGA-A score of 0 at each study visit after baseline, stratified by initial CTP regimen.

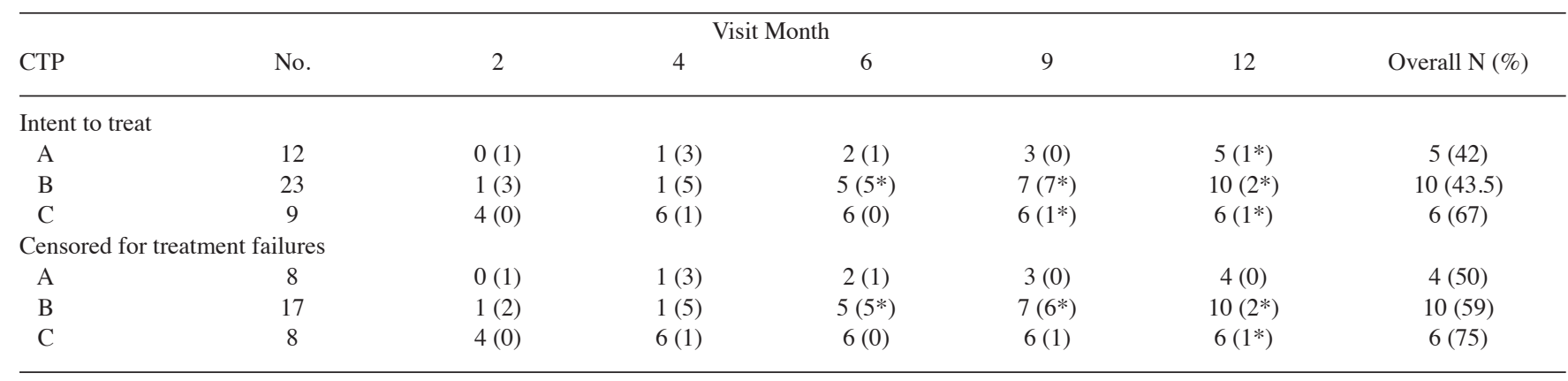

To track improvement in PGA-A scores across visits, the total no. patients who had achieved a PGA-A of 0 at each study visit after baseline were counted. The total number of patients who had achieved a PGA-A score of 0 by this visit are listed and the number of patients who were missing at each visit and had not yet reached a PGA-A score of 0 is noted in parantheses. Followup visits were specified to occur within 1 month of target time of 2 months, 4 months, 6 months, 9 months, and 12 months. The top panel shows the values for all patients who were receiving systemic immunosuppressive treatment at last study visit based upon the initially prescribed CTP; the bottom panel excludes the 11 patients who experienced treatment failure. A number of patients in CTP B missed their 4-, 6-, and/or 9-month visits; however, at 12-month visit, the only missing data were due to censoring. In CTP A, data from 1 patient was censored at the 12-month visit because of dropout due to treatment failure; this patient is represented only in the top panel. In CTP B, data from 2 patients were censored at the 6-month visit, and in CTP C data from 1 patient was censored at the 9-month visit, because of treatment discontinuation secondary to adverse events.

Table 4B. The total number of patients who achieved a PGA-A score of 0 at each study visit after baseline, stratified by baseline PGA-A score.

\begin{tabular}{|c|c|c|c|c|c|c|c|}
\hline \multicolumn{8}{|c|}{ Visit Month } \\
\hline Baseline PGA-A & No. & 2 & 4 & 6 & 9 & 12 & Overall No. (\%) \\
\hline \multicolumn{8}{|l|}{ Intent to treat } \\
\hline $3-4$ & 18 & $2(1)$ & $4(6)$ & $5(2)$ & $8(2)$ & $10(0)$ & $10(56)$ \\
\hline $5-7$ & 16 & $3(3)$ & $3(1)$ & $5(1)$ & $5(3)$ & $6(1 *)$ & $7(44)$ \\
\hline $8+$ & 6 & $0(0)$ & $0(1)$ & $0\left(3^{*}\right)$ & $1\left(3^{*}\right)$ & $1\left(3^{*}\right)$ & $1(13)$ \\
\hline \multicolumn{8}{|c|}{ Censored for treatment failures } \\
\hline $3-4$ & 14 & $2(1)$ & $4(6)$ & $5(2)$ & $8(2)$ & $9(0)$ & $9(64)$ \\
\hline $5-7$ & 10 & $3(2)$ & $3(1)$ & $5(1)$ & $5(2)$ & $6(0)$ & $7(70)$ \\
\hline $8+$ & 5 & $0(0)$ & $0(1)$ & $0\left(3^{*}\right)$ & $1(3 *)$ & $1\left(3^{*}\right)$ & $1(13)$ \\
\hline
\end{tabular}

The top panel shows the values for all patients who were receiving systemic immunosuppressive treatment at last study visit based upon the initially prescribed CTP; the bottom panel excludes the 11 patients who were treatment failures. Data from 1 patient in the baseline PGA-A 5-7 score group was censored at the 12-month visit in the top panel; data from 3 patients in the baseline PGA-A 8+ score group were censored at the 6-month visit in both panels. * Visit data censored because of AE requiring deviation from a CTP regimen $(n=3)$ or treatment failure $(n=1)$. CTP: consensus treatment plan; PGA-A: physician's global assessment of disease activity; AE: adverse events.

scores for patients in CTP C may reflect their having less extensive skin disease (Table 2).

Patients were stratified into 4 levels of baseline PGA-A scores: $1-2,3-4,5-7$, and $>8$, to determine whether the baseline PGA-A score affected the likelihood of reaching PGA-A $=0$. Higher baseline PGA-A scores were associated with both lower likelihoods and slower rates of achieving PGA-A $=0$. Two-thirds of non-TF patients who had a baseline PGA-A score of $<7$ reached 0 within 12 months, compared to $13 \%$ of patients with a PGA-A of 8 or above $(\mathrm{p}=0.045$; Table 4B).

Extent of TF. Eleven patients were considered to have experienced TF and received additional treatment: IV CS $(\mathrm{n}=10)$ and/or mycophenolate mofetil (MMF; $\mathrm{n}=8)$. A larger percentage of patients experienced TF in CTP A $(\mathrm{n}=4,33 \%)$ and CTP B $(\mathrm{n}=6,25 \%)$ than CTP C $(\mathrm{n}=1$,
$12.5 \%$ ), but these differences were not significant (Figure 1B). Four patients enrolled in CTP A (MTX monotherapy) received IV CS, and 6 patients in CTP B received longer IV CS courses; 1 also received oral CS. Eight patients (1 CTP A, 6 CTP B , 1 CTP C) received MMF, all concurrently with MTX. Median time to TF was at the 4-month visit (median 116 days); range 2- to 9-month visit (97-302 days).

At the last visit, most patients who experienced TF were rated as having a moderate level of improvement ( $\Delta$-activity, $\mathrm{n}=6,54.5 \%$ ) versus the major level of improvement given to most non-TF patients $(\mathrm{n}=18,56.3 \%)$. PGA-A, mLoSSI, and LSCAM scores decreased from baseline to last visit in patients who experienced TF (Table 5), but these patients did not experience as much improvement as non-TF patients. At last visit, they had higher PGA-A, mLoSSI, and LSCAM scores than non-TF patients $(\mathrm{p}=0.011,0.003$, 
Table 5. Characteristics of patients who experienced treatment failure (TF).

\begin{tabular}{|c|c|c|c|c|}
\hline \multicolumn{2}{|c|}{ Characteristics } & No TF & $\mathrm{TF}$ & $\mathrm{p}$ \\
\hline \multicolumn{2}{|c|}{ No. patients } & 33 & 11 & \\
\hline \multicolumn{2}{|l|}{ CTP A } & $8(24)$ & $4(36)$ & NS \\
\hline \multicolumn{2}{|l|}{ СТP B } & $17(51.5)$ & $6(55)$ & NS \\
\hline \multicolumn{2}{|l|}{ СТP C } & $8(24)$ & $1(9)$ & NS \\
\hline \multicolumn{2}{|c|}{ Age at enrollment, yrs, median (IQR) } & $13(9.4-14.1)$ & $12.1(9.8-15.1)$ & NS \\
\hline \multicolumn{2}{|c|}{ Age of onset, yrs, median (IQR) } & $9.03(5.7-10.6)$ & $10.1(9.2-13.5)$ & 0.076 \\
\hline \multicolumn{2}{|c|}{$\begin{array}{l}\text { Disease duration at enrollment, mos, } \\
\text { median (IQR) }\end{array}$} & $24(6.50-76.0)$ & $10(6-13.0)$ & NS \\
\hline \multicolumn{2}{|l|}{ Female } & $23(70)$ & $8(73)$ & NS \\
\hline \multicolumn{2}{|l|}{ Race } & & & 0.038 \\
\hline \multicolumn{2}{|l|}{ White } & $32(97)$ & $9(82)$ & \\
\hline \multicolumn{2}{|l|}{ Asian } & 0 & $2(18)$ & \\
\hline \multicolumn{2}{|l|}{ Mixed } & $1(3)$ & 0 & \\
\hline \multicolumn{2}{|l|}{ Hispanic } & $7(21)$ & $2(18)$ & NS \\
\hline \multicolumn{2}{|l|}{ Subtype } & & & 0.010 \\
\hline \multicolumn{2}{|c|}{ Circumscribed superficial } & $3(9)$ & 0 & \\
\hline \multicolumn{2}{|c|}{ Circumscribed deep } & $1(3)$ & $1(9)$ & \\
\hline \multicolumn{2}{|c|}{ Linear scleroderma } & $25(76)$ & $3(27)$ & \\
\hline \multicolumn{2}{|c|}{ Generalized morphea } & 0 & $1(9)$ & \\
\hline \multicolumn{2}{|c|}{ Pansclerotic morphea } & 0 & $1(9)$ & \\
\hline \multicolumn{2}{|c|}{ Mixed morphea } & $4(12)$ & $5(45.5)$ & \\
\hline \multicolumn{2}{|c|}{ Head involvement } & $15(45.5)$ & $2(18)$ & NS \\
\hline \multicolumn{2}{|c|}{ Trunk involvement } & $12(36)$ & $8(73)$ & 0.036 \\
\hline \multicolumn{2}{|c|}{ Limb involvement } & $16(48.5)$ & $9(82)$ & 0.053 \\
\hline \multicolumn{2}{|c|}{ Affected anatomic sites, median (IQR) } & $2(1-3)$ & $4(2-8)$ & 0.096 \\
\hline \multicolumn{2}{|c|}{ Extracutaneous involvement } & $22(67)$ & $11(100)$ & 0.027 \\
\hline Growth $\mathrm{c}$ & & $14(42.4)$ & $7(63.6)$ & NS \\
\hline Joint inv & & $10(30)$ & $10(91)$ & 0.005 \\
\hline PGA-A & Baseline, median (IQR) & $4(3-6)$ & $5(4,7)$ & NS \\
\hline & Last, median (IQR) & $0(0-1)^{* *}$ & $2(1,3) * *$ & 0.011 \\
\hline mLoSSI & Baseline, median (IQR) & $8(4-10)$ & $5(4,9)$ & NS \\
\hline & Last, median (IQR) & $0(0-1)^{* *}$ & $2(1,4)^{* *}$ & 0.003 \\
\hline LSCAM & Baseline, median (IQR) & $7(4-12)$ & $8(6,23)$ & NS \\
\hline & Last, median (IQR) & $1(0-2)^{* *}$ & $4(3,10)^{* *}$ & $<0.001$ \\
\hline LoSDI & Baseline, median (IQR) & $7(3-13)$ & $14(2,23)$ & NS \\
\hline & Last, median (IQR & $7(4-11)$ & $12(4,26)$ & NS \\
\hline LSDam & Baseline, median (IQR) & $9(5-16)$ & $16(4,34)$ & NS \\
\hline & Last, median (IQR) & $8(5-11)$ & $12(6,35)^{*}$ & NS \\
\hline PGA-D & Baseline, median (IQR) & $4(3-5)$ & $5(4-7)$ & NS \\
\hline & Last, median (IQR) & $3(2-4)$ & $5(3-7)$ & 0.003 \\
\hline ANA pos & & $14(52)$ & $9(82)$ & 0.087 \\
\hline Family h & hatic disease & $11(34)$ & $7(64)$ & 0.090 \\
\hline
\end{tabular}

Values are $\mathrm{n}(\%)$ unless otherwise specified. $\mathrm{P}$ values for differences between patients who experienced TF versus those who did not are shown in last column. Significant $\mathrm{p}$ values are in bold face; values that approached significance are in italics. Significant changes in scores from baseline to last visit are indicated in "No TF" and "TF" columns: $* \mathrm{p}<0.05$; ** $\mathrm{p}<0.001$. The 11 patients who experienced TF were compared to the 33 other patients who remained on their initial CTP treatment and did not require additional treatment. Head, trunk, limb involvement refers to the no. patients who had an LS lesion in that anatomic site. The no. anatomic sites is based upon the divisions used for the LS Cutaneous Activity measure; the body is divided into 19 sites. The p values on the Race and Subtype rows refer to the significant difference found in the distribution of all of the listed races or subtypes, respectively. Also found significant were frequency of linear scleroderma $(\mathrm{p}=0.0089)$, mixed morphea $(p=0.0305)$, and mixed, generalized, and pansclerotic morphea together $(p=0.002)$; generalized and pansclerotic morphea together approached significance $(\mathrm{p}=0.058)$. ANA: antinuclear antibody; CTP: consensus treatment plan; IQR: interquartile range; LoSDI: Localized Scleroderma Damage Index; LSCAM: localized scleroderma cutaneous activity measure; LSDam: localized scleroderma cutaneous damage measure; mLoSSI: modified Localized Scleroderma Severity Index; NS: nonsignificant; PGA-A: physician's global assessment of disease activity; PGA-D: PGA of disease damage. 
$<0.001$, respectively; Table 5). At the last visit, PGA-D scores were also higher in TF than non-TF patients $(\mathrm{p}=0.003$; Table 5). Patients who experienced TF were more likely to be non-white race and to have mixed morphea, generalized morphea, pansclerotic morphea, ECI, joint involvement, and truncal lesions (Table 5). There was a trend toward significance for limb involvement, more extensive skin disease, older age of disease onset, ANA positivity, and family history of a rheumatic disease $(\mathrm{p}<0.1$; Table 5).

\section{DISCUSSION}

To our knowledge, this is the first prospective comparative effectiveness study of 3 different MTX-based regimens for juvenile LS. The study aims were to assess the safety and feasibility of the 3 standardized regimens and evaluate our assessment tools. Our prior survey of North American pediatric rheumatologists demonstrated the need for comparative effectiveness studies, because responders were almost equally divided on their choice of these 3 MTX-based $\mathrm{CTP}^{23}$. Our investigators similarly showed distinct treatment preferences, with half the sites choosing a single CTP to treat all their study patients ${ }^{24}$. Because the physician and parent/patient jointly selected the treatment CTP, some of these choices reflect family preference with at least 3 families opting for MTX monotherapy over their physician's recommendation for a CS-associated CTP.

All 3 CTP were found to be safe, generally well tolerated, and effective, with over half the patients having PGA-A $=0$ by their last visit. However, only about half completed the CTP they initially started; dropouts and $\mathrm{AE}$ accounted for $52 \%$ of the deviations, and $\mathrm{TF}$ the remainder. The frequencies of AE for MTX and CS were similar across the CTP. Most AE were managed by supportive care or brief pauses in treatment. Six patients (14\%) had their CTP regimen changed because of intolerance. This frequency is higher than was reported in the CS-based randomized controlled trial of MTX $^{15}$, possibly reflecting higher MTX dose $\left(1 \mathrm{mg} / \mathrm{kg} /\right.$ dose vs $15 \mathrm{mg} / \mathrm{m}^{2}$, potentially double for a small child) or different route of administration. Other explanations are differences in CS regimen including the route, longer and higher oral prednisone dose, and/or patient characteristics.

Most who experienced TF received mycophenolate mofetil (MMF), all in combination with MTX. Our group had previously developed an MMF-based CTP that allowed for use of MMF with or without $\mathrm{MTX}^{24}$, but it was not included in the current study. The high frequency of CTP failures suggests that additional standardized treatment regimens are likely needed for comparative effectiveness studies, including evaluating the efficacy of MMF separate from MTX, and examining biologics or other disease-modifying antirheumatic drugs.

Several physician scores and skin activity measures were used to assess treatment response. We found limitations in the physician assessment of overall disease status compared to baseline ( $\Delta$-disease status) because it encompasses damage as well as activity status. Three patients developed worsening of extracutaneous features by their last visit, and although all were rated as improved by $\Delta$-activity status, all were scored as having a worsened disease status compared to baseline. These patients demonstrate that damage can progress despite effective control of disease activity. We therefore recommend that activity and damage be separately assessed for juvenile LS, with initial treatment efficacy evaluated primarily by activity measures.

We detected differences between subpopulations, suggesting that our assessments are sensitive enough to use in comparative effectiveness studies. Our LSCAM includes more variables than are found in the mLoSSI, the model for our measure. Both measures detected significant changes in skin scores between first and last visit; the LSCAM also detected differences between CTP and showed a wider range of scores in the TF patients than did the mLoSSI. Higher baseline PGA-A scores were associated with a slower and lower rate of response, especially if the baseline PGA-A score was 8 or higher. Our analysis of the TF patients identified additional variables associated with poorer response including truncal lesions, ECI, joint involvement, non-white race, and mixed morphea subtype. More extensive skin involvement, limb involvement, and ANA positivity approached significance. Further evaluation is needed to determine whether some of these variables are confounders that should be considered when designing treatment trials or evaluating response.

Because this was a pilot study, it was not powered for determining the relative efficacy of the CTP. There was a trend toward a faster and higher rate of response in one of the CTP groups, which may reflect differences in disease severity and pattern between groups. Patients in the CTP associated with poorer response had higher LSCAM scores at baseline, more extensive skin disease, and higher frequency of mixed morphea subtype and growth disturbances $(\mathrm{p}<0.05)$, as well as a greater likelihood of joint involvement $(p<0.1)$. Some of these variables were also identified in patients who experienced TF, suggesting they may represent prognostic features that are confounding the relationship between treatment and response. In a larger sample, case control methods could be used to match subjects according to variables associated with disease severity/activity to more effectively evaluate the efficacy of the CTP without selection bias.

Study limitations include the large variation in time of the "12-month" study visit, with several patients seen outside of the recommended window of $12 \pm 1$ month. This is to be expected in prospective observational studies, where visits are completed according to requirements for care rather than the more rigorous schedule of standardized treatment trials.

Personal non-commercial use only. The Journal of Rheumatology Copyright @ 2020 . All rights reserved. 
Another limitation is the absence of serological biomarkers to identify or quantify disease activity. We performed several clinical assessments in tandem to improve our accuracy of clinical scoring. However, physicians may be biased toward a given CTP efficacy, so use of assessors blinded to the chosen CTP may be advisable.

Our study findings add substantially to the understanding of treatment strategies for juvenile LS patients. All 3 CTP were found beneficial, yielding tangible and significant improvements in disease activity. Our cutaneous activity measure and physician assessments could identify both changes during the course of treatment and differences in response between some patient subsets. More studies are needed to assess the reliability, validity, and performance of our LSCAM measure compared to mLoSSI. Further study is needed to determine if these clinical outcome measures are sensitive enough to detect differences in relative efficacy between treatments. Studies are also needed to develop biomarkers to enhance monitoring of response.

The widespread occurrence of ECI in our cohort (> 70\%) demonstrates that juvenile LS is a serious disease with a high potential for severe morbidity. All patients who experienced TF had ECI; at the end of the study they had higher LSCAM and physician global damage scores. In addition, despite nearly all patients having a marked reduction in disease activity level, damage scores did not improve, and in some patients worsened. Our findings suggest that patients with a larger disease burden (skin and other tissues) are at risk for more damage and require more treatment for disease control. It may be worthwhile considering treat-to-target strategies for juvenile LS, as has been proposed for rheumatoid arthritis ${ }^{39}$. Larger, more robust comparative effectiveness studies are needed to objectively identify optimal treatment strategies that minimize disease burden, medication intolerance, and damage progression. Findings from this study should help inform the development of such studies. We expect an iterative process that will improve our ability to optimize therapy and longterm outcome for these patients.

\section{ACKNOWLEDGMENT}

The authors thank Laura Schanberg for her key help and support of this project. We also thank the following CARRA Registry site principal investigators and research coordinators: E. Anderson, H. Benham, B. Feldman, K. Francis, I. Goh, J. Jaquith, K. Schollaert-Fitch, C. Smith, J. Weiss, J. Wooton, and all study staff who helped recruit patients for the CARRA Registry. We thank the patients and families who participated in our study. We gratefully acknowledge Marilynn Punaro and Brian Feldman for their sage advice and support of this project. We thank Xiaohu Li for initial help with data analysis; Maria Carputo and Danielle Wolfe for help with data entry; and Justine Griswold, Mary Ellen Riordan, Maria Carrollo, and Jeannette Haugh for help with study coordination, data management, and meeting planning. We thank Jane Winsor and Kelly Mieszkalski for their expertise and help with the CARRA Legacy Registry.

\section{REFERENCES}

1. Torok KS, Li SC, Jacobe HM, Taber SF, Stevens AM, Zulian F, et al. Immunopathogenesis of pediatric localized scleroderma. Front Immunol 2019;10:908.

2. Jacobe H, Ahn C, Arnett F, Reveille J. Major histocompatibility complex class I and class II alleles may confer susceptibility to or protection against morphea. Arthritis Rheumatol 2014;66:3170-7.

3. Condie D, Grabell D, Jacobe H. Comparison of outcomes in adults with pediatric-onset morphea and those with adult-onset morphea: a cross-sectional study from the morphea in adults and children cohort. Arthritis Rheumatol 2014;66:3496-504.

4. Li SC. Scleroderma in children and adolescents: localized scleroderma and systemic sclerosis. Pediatr Clin North Am 2018;65:757-81.

5. Peterson L, Nelson A, Su WP, Mason T, O'Fallon WM, Gabriel SE. The epidemiology of morphea (localized scleroderma) in Olmstead County 1960-1993. J Rheumatol 1997;24:73-80.

6. Weibel L, Laguda B, Atherton D, Harper JI. Misdiagnosis and delay in referral of children with localized scleroderma. Br J Dermatol 2011;165:1308-13.

7. Piram M, McCuaig CC, Saint-Cyr C, Marcoux D, Hatami A, Haddad E, et al. Short- and long-term outcome of linear morphoea in children. Br J Dermatol 2013;169:1265-71.

8. Saxton-Daniels S, Jacobe HT. An evaluation of long-term outcomes in adults with pediatric-onset morphea. Arch Dermatol 2010;146:1044-45.

9. Wu E, Li S, Torok K, Virkud Y, Fuhlbrigge R, Rabinovich E, et al; Childhood Arthritis and Rheumatology Research Alliance (CARRA) Legacy Registry Investigators. Baseline description of the juvenile localized scleroderma subgroup from the Childhood Arthritis and Rheumatology Research Alliance legacy registry. ACR Open Rheumatol 2019;1:119-24.

10. Li SC, Feldman BM, Higgins GC, Haines KA, Punaro MG, O’Neil KM. Treatment of pediatric localized scleroderma: results of a survey of North American pediatric rheumatologists. J Rheumatol 2010;37:175-81.

11. Zulian F, Culpo R, Sperotto F, Anton J, Avcin T, Baildam EM, et al. Consensus-based recommendations for the management of juvenile localised scleroderma. Ann Rheum Dis 2019;78:1019-24.

12. Kreuter A, Krieg T, Worm M, Wenzel J, Moinzadeh P, Kuhn A, et al. German guidelines for the diagnosis and therapy of localized scleroderma. J Dtsch Dermatol Ges 2016;14:199-216.

13. Mertens J, Seyger M, Thurlings R, Radstake T, de Jong EMGJ. Morphea and eosinophilic fasciitis: an update. Am J Clin Dermatol 2017;18:491-512.

14. Kroft E, Creemers M, Van Den Hoogen F, Boezeman J, De Jong E. Effectiveness, side-effects and period of remission after treatment with methotrexate in localized scleroderma and related sclerotic skin diseases: an inception cohort study. Br J Dermatol 2009;160:1075-82.

15. Zulian F, Martini G, Vallongo C, Vittadello F, Falcini F, Patrizi A, et al. Methotrexate treatment in juvenile localized scleroderma: a randomized, double-blind, placebo-controlled trial. Arthritis Rheum 2011;63:1998-2006.

16. Torok KS, Arkachaisri T. Methotrexate and corticosteroids in the treatment of localized scleroderma: a standardized prospective longitudinal single-center study. J Rheumatol 2012;39:286-94.

17. Weibel L, Sampaio MC, Visentin MT, Howell KJ, Woo P, Harper JI. Evaluation of methotrexate and corticosteroids for the treatment of localized scleroderma (morphoea) in children. Br J Dermatol 2006;155:1013-20.

18. Uziel Y, Feldman B, Krafchik B, Yeung R, Laxer R. Methotrexate and corticosteroid therapy for pediatric localized scleroderma. J Pediatr 2000;136:91-5.

19. Fitch PG, Rettig P, Burnham JM, Finkel TH, Yan AC, Akin E, et al.

Personal non-commercial use only. The Journal of Rheumatology Copyright @ 2020 . All rights reserved 
Treatment of pediatric localized scleroderma with methotrexate. J Rheumatol 2006;33:609-14

20. Cox D, O'Regan G, Collins S, Byrne A, Irvine A, Watson R. Juvenile localised scleroderma: a retrospective review of response to systemic treatment. Ir J Med Sci 2008;177:343-6.

21. Mertens J, van den Reek J, Kievit W, van de Kerkhof P, Thurlings $\mathrm{R}$, Radstake $\mathrm{T}$, et al. Drug survival and predictors of drug survival for methotrexate treatment in a retrospective cohort of adult patients with localized scleroderma. Acta Derm Venereol 2016;96:943-47.

22. Kreuter A, Gambichler T, Breuckmann F, Rotterdam S, Freitag M, Stuecker M, et al. Pulsed high-dose corticosteroids combined with low-dose methotrexate in severe localized scleroderma. Arch Dermatol 2005;141:847-52.

23. Li SC, Torok KS, Pope E, Dedeoglu F, Hong S, Jacobe HT, et al; Childhood Arthritis and Rheumatology Research Alliance (CARRA) Localized Scleroderma Workgroup. Development of consensus treatment plans for juvenile localized scleroderma: a roadmap toward comparative effectiveness studies in juvenile localized scleroderma. Arthritis Care Res 2012;64:1175-85.

24. Li SC, Fuhlbrigge RC, Laxer RM, Pope E, Ibarra MF, Stewart K, et al; CARRA Registry Investigators. Developing comparative effectiveness studies for a rare, understudied pediatric disease: lessons learned from the CARRA juvenile localized scleroderma consensus treatment plan pilot study. Pediatr Rheumatol Online J 2019;17:43.

25. Laxer RM, Zulian F. Localized scleroderma. Curr Opin Rheumatol 2006;18:606-13.

26. Czirják L, Nagy Z, Aringer M, Riemekasten G, Matucci-Cerinic M, Furst D, et al. The EUSTAR model for teaching and implementing the modified Rodnan skin score in systemic sclerosis. Ann Rheum Dis 2007;66:966-9.

27. U.S. Department of Health and Human Services. Common terminology criteria for adverse events version (CTCAE), version 4. [Internet. Accessed March 6, 2020.] Available from: evs.nci. nih.gov/ftp1/CTCAE/CTCAE_4.03/CTCAE_4.03_2010-06-14_ QuickReference_8.5x11.pdf

28. Arkachaisri T, Vilaiyuk S, Li S, O'Neil KM, Pope E, Higgins GC, et al; Localized Scleroderma Clinical and Ultrasound Study Group. The localized scleroderma skin severity index and physician global assessment of disease activity: a work in progress toward development of localized scleroderma outcome measures. J Rheumatol 2009;36:2819-29.
29. Arkachaisri T, Vilaiyuk S, Torok KS, Medsger TA Jr. Development and initial validation of the localized scleroderma skin damage index and physician global assessment of disease damage: a proof-of-concept study. Rheumatology 2010;49:373-81.

30. Arkachaisri T, Pino S. Localized scleroderma severity index and global assessments: a pilot study of outcome instruments. J Rheumatol 2008;35:650-7.

31. Li SC, Li X, Pope E, Stewart K, Higgins GC, Rabinovich CE, et al. New features for measuring disease activity in pediatric localized scleroderma. J Rheumatol 2018;45:1680-8.

32. Zulian F, Vallongo C, Woo P, Russo R, Ruperto N, Harper J, et al; Juvenile Scleroderma Working Group of the Pediatric Rheumatology European Society (PRES). Localized scleroderma in childhood is not just a skin disease. Arthritis Rheum 2005; 52:2873-81.

33. Lis-Swiety A, Janicka I, Skrzypek-Salamon A, Brzezinska-Wcislo L. A systematic review of tools for determining activity of localized scleroderma in paediatric and adult patients. J Eur Acad Dermatol Venereol 2017;31:30-7.

34. Singh G, Athreya B, Fries J, Goldsmith D. Measurement of health status in children with juvenile rheumatoid arthritis. Arthritis Rheum 1994;37:1761-9.

35. Varni J, Seid M, Kurtin PS. PedsQL 4.0: reliability and validity of the Pediatric Quality of Life Inventory version 4.0 generic core scales in healthy and patient populations. Medical Care 2001;39:800-12.

36. Varni J, Seid M, Smith Knight T, Burwinkle T, Brown J, Szer IS. The PedsQL in pediatric rheumatology: reliability, validity, and responsiveness of the Pediatric Quality Of Life Inventory Generic Core Scales and Rheumatology Module. Arthritis Rheum 2002;46:714-25.

37. Varni J, Sherman S, Burwinkle T, Dickinson P, Dixon P. The PedsQL Family Impact Module: preliminary reliability and validity. Health Quality Life Outcomes 2004;2:55.

38. Lewis-Jones M, Finlay A. The Children's Dermatology Life Quality Index (CDLQI): initial validation and practical use. Br J Dermatol 1995;132:942-9.

39. Smolen J, Breedveld F, Burmester G, Bykerk V, Dougados M, Emery P, et al. Treating rheumatoid arthritis to target: 2014 update of the recommendations of an international task force. Ann Rheum Dis 2016;75:3-15. 